\title{
PRODUÇÃO DE BIODIESEL POR TRANSESTERIFICAÇÃO UTILIZANDO CATALISADOR HETEROGÊNEO (KOH/Al $\left.{ }_{2} \mathrm{O}_{3}\right)$
}

\author{
A. D. GONDIM*, M. F. V. SANTOS, T. P. OLIVEIRA, C. G. D. PEIXOTO, A. C. M. BATISTA, V. J FERNANDES JR. \\ Universidade Federal do Rio Grande de Norte \\ amandagondim.ufrn@gmail.com*
}

Submetido 10/10/2016 - Aceito 16/02/2017

DOI: $10.15628 /$ holos.2017.5195

\section{RESUMO}

O biodiesel consiste de um combustível biodegradável derivado de óleos vegetais e gorduras animais, formado por ésteres de ácidos graxos, ésteres de alquila (metila, etila ou propila) de ácidos carboxílicos de cadeia longa. O processo convencional para sua produção ocorre pela transesterificação de óleos vegetais com álcool na presença de um catalisador. O catalisador mais utilizado no processo de transesterificação é o catalisador homogêneo, $\mathrm{KOH}$, que apresenta grande dificuldade de recuperação do mesmo, portanto foi estudado neste trabalho um catalisador heterogêneo $\left(\mathrm{KOH} / \mathrm{Al}_{2} \mathrm{O}_{3}\right)$. Deste modo, este trabalho tem como objetivo e obter o catalisador e caracterizar, além de aplicar o catalisador na reação de transesterificação de óleo de algodão em busca das melhores condições de reações. Os produtos obtidos foram avaliados por densidade, viscosidade, análise térmica (TG/DTG) e espectroscopia na região do infravermelho por transformada de Fourier (FTIR). Para a confirmação da obtenção do catalisador foi utilizada a técnica como por difratograma de raio $X$ (DRX), microscópico eletrônico de varredura (MEV-EDS), FTIR e TG/DTG . Os biodiesel obtido através da reação de transesterificação na presença de $\mathrm{KOH} / \mathrm{Al}_{2} \mathrm{O}_{3}$ apresentaram resultados de densidade e viscosidade de acordo com Resolução ANP 45/2014. O biodiesel que foi obtido com um tempo reacional de $5 \mathrm{~h}$ apresentou maior percentual de conversão $(94,5 \%)$.

PALAVRAS-CHAVE: Biodiesel, $\mathrm{KOH} / \mathrm{Al}_{2} \mathrm{O}_{3}$, Transesterificação, Termogravimetria, Catalise heterogênea.

\section{BIODIESEL PRODUCTION BY TRANSESTERIFICATION USING HETEROGENEOUS CATALYST $\left(\mathrm{KOH} / \mathrm{Al}_{2} \mathrm{O}_{3}\right)$}

\begin{abstract}
Biodiesel is a biodegradable fuel derived from vegetable oils and animal fats composed of esters of fatty acids, alkyl esters (methyl, ethyl or propyl) long-chain carboxylic acids. The conventional process for production of biodiesel occurs by transesterification of vegetable oils with alcohol in the presence of a catalyst. The most commonly used catalyst in transesterification process is a homogeneous catalyst, $\mathrm{KOH}$, which has great difficulty in recovering same, so was used in this study a heterogeneous catalyst $\left(\mathrm{KOH} / \mathrm{Al}_{2} \mathrm{O}_{3}\right)$. Thus, this paper aims to obtained the catalyst and characterize, in addition to applying the catalyst of cottonseed oil in the transesterification reaction in search of better
\end{abstract}

conditions of reaction. The products obtained were evaluated by density, viscosity, thermal analysis (TG/DTG) and Fourier Transform Infrared (FTIR) spectroscopy. For confirmation of obtaining the catalyst was used the technique as by $X$ ray diffraction (XRD), scanning electron microscopy (SEM-EDS), FTIR and TG/DTG. Biodiesel obtained by the transesterification reaction in the presence of $\mathrm{KOH} / \mathrm{Al}_{2} \mathrm{O}_{3}$ shows results of density and viscosity according to ANP Resolution 45/2014. The biodiesel was obtained with a reaction time of $5 \mathrm{~h}$ showed higher conversion percentage (94.5\%).

PALAVRAS-CHAVE: Biodiesel, $\mathrm{KOH} / \mathrm{Al}_{2} \mathrm{O}_{3}$, transesterification, Thermogravimetry, Heterogeneous Catalyst. 


\section{INTRODUÇÃO}

A maior parte de toda a energia consumida no mundo provém do petróleo, do carvão e do gás natural. Mas, o mundo vem buscando por novas alternativas da matriz energética e com isso as fontes naturais de biomassa são uma alternativa viável. Portanto, cresce o incentivo para desenvolvimento de tecnologias que permitam utilizar fontes de energia renováveis, ecologicamente correta e viável economicamente.

O biodiesel consiste de um combustível biodegradável derivado de óleos vegetais e gorduras animais, formado por ésteres de ácidos graxos, ésteres de alquila (metila, etila ou propila) de ácidos carboxílicos de cadeia longa (SILVA, 2015; OLIVEIRA, 2006). O biodiesel tem características excelentes como o diesel e, portanto, pode se utilizados em motor de compressão com pequena ou nenhuma modificação ( $\mathrm{Xi}$ e $\mathrm{Li}, 2006)$. O processo convencional para sua produção ocorre pela transesterificação de óleos vegetais com álcool na presença de um catalisador homogêneo (EVANGELISTA, 2011). O KOH é bastante utilizado como catalisador homogêneo, mas apresenta grande dificuldade de recuperação do mesmo, portanto, este trabalho propõe um catalisador heterogêneo.

A utilização de catalisadores heterogêneos tem algumas vantagens como: não é corrosivo; ambiente favorável; apresenta menos problemas na eliminação; fácil de separar a partir de produtos líquidos; pode ser concebido para ter maior atividade, seletividade e tempo de vida. Na catálise heterogênea, o catalisador é formado por uma fase ativa e suporte, geralmente inerte. Nesse contexto, a alumina por possui área superficial elevada é bastante utilizada como suporte de catalisadores (CORDEIRO et al., 2011).

Para a reação de transesterificação é interessante que o catalisador possua sítios básicos e a presença de metais alcalinos e alcalinos terrosos confere ao suporte a sítios básicos de Lewis. Portanto, este trabalho propõe a síntese e caracterização de um catalisador heterogêneo $\left(\mathrm{KOH} / \mathrm{Al}_{2} \mathrm{O}_{3}\right)$ e a avaliação da sua aplicação durante a reação de transesterificação de óleo de algodão, juntamente com o melhor tempo reacional.

\section{REVISÃO BIBLIOGRÁFICA}

A produção e o uso do biodiesel no Brasil são incentivados pelo governo, pois propiciam o desenvolvimento de uma fonte energética sustentável sob os aspectos ambiental, econômico e social. Além de contribuir com a redução das importações de óleo diesel, gerando divisas para o País (ANP, 2016). No Brasil, o biodiesel é adicionado ao diesel (BX), assim sua utilização é compulsória.

O biodiesel pode ser produzido pela reação de transesterificação ou esterificação (presença de ácidos graxos livres) de óleos vegetais e/ou gorduras animais com álcool de cadeia curta e na presença de catalisador ácido ou básico, que pode ser homogêneo ou heterogêneo. Os catalisadores enzimáticos também podem ser utilizados para produção de biodiesel (CORDEIRO et. al. 2011). Para que a reação transesterificação ocorrer com sucesso e necessário controlar as condições reacionais, ou seja, tipo do álcool, razão molar óleo: álcool, temperatura e tempo de da reação, tipo do catalisado e a quantidade utilizada. 
Os catalisadores para produção de biodiesel mais estudada são os que apresentam sítios ácidos e/ou básicos de Lewis. Para reação de esterificação são mais eficazes catalisadores com presença de sitos ácidos e para transesterificação os sítios básicos (YAN et, al. 2009).

Apesar de catalisadores homogênios básicos ( $\mathrm{NaOH}$ e $\mathrm{KOH})$ apresentarem melhores teores de conversões, muitas pesquisas estão sendo focadas na busca por catalisadores heterogêneos, pois estes podem ser facilmente recuperados do meio reacional, como também, são materiais ambientalmente corretos. Os catalisadores heterogêneos são formados de um suporte e uma fase ativa. $\mathrm{O}$ uso do suporte é essencial para que o catalisador não se transfira para a fase líquida da reação, sendo a alumina ou óxido de alumínio $\left(\mathrm{Al}_{2} \mathrm{O}_{3}\right)$ bastante utilizado como catalisador, absorvente ou suporte por apresentar uma área específica elevada (EVANGELISTA, 2011).

Dentro deste contexto, são encontrados na literatura alguns trabalhos utilizando catalisadores heterogêneo para transesterificação de óleos vegetais suportados em alumina impregnada com $\mathrm{K}_{2} \mathrm{CO}_{3}$ (GAMA et, al., 2015), KF (BOZ et, al. 2009) , $\mathrm{CaO}$ (UMDU e SEKER, 20120 , MgZnO (OLUTOYE e HAMEED, 2013), $\mathrm{LiNO}_{3}($ ISTADI et. al., 2010), entre outros.

Noiroj (2009) estudou o $\mathrm{KOH}$ suportado em $\mathrm{Al}_{2} \mathrm{O}_{3}$ e $\mathrm{NaY}$ zeólita como catalisador heterogêneo na transesterificação de óleo de palma. Ele produziu uma série de catalisadores de $\mathrm{KOH} / \mathrm{Al}_{2} \mathrm{O}_{3}$ com diferentes massas de $\mathrm{KOH}(10,15,20,25,30$ e 35\%). Os catalisadores foram preparados por impregnação de um suporte de $\mathrm{Al}_{2} \mathrm{O}_{3}$ com uma solução aquosa de $\mathrm{KOH}$. Os catalisadores preparados foram secos num forno a 110 으 durante $24 \mathrm{~h}$ e foram calcinados a 500 o $C$ durante 3 horas. Verificou-se que a nova fase ativa de $\mathrm{K}_{2} \mathrm{O}$ foi a causa da elevada atividade catalítica e basicidade do catalisador uma vez que, quando se aumenta a carga de $\mathrm{KOH}$ a $15-25 \%$, observou-se a fase de $\mathrm{K}_{2} \mathrm{O}$ e o rendimento de biodiesel foi aumentado, obtendo $87,5 \%$ ésteres metílicos utilizando o catalisador de $20 \% \mathrm{~m} / \mathrm{m} \mathrm{KOH} / \mathrm{Al}_{2} \mathrm{O}_{3}$ e $91,07 \%$ utilizando o catalisadores $25 \% \mathrm{~m} / \mathrm{m} \mathrm{KOH} / \mathrm{Al}_{2} \mathrm{O}_{3}$ e $10 \% \mathrm{~m} / \mathrm{m} \mathrm{KOH} / \mathrm{NaY}$ a uma temperatura de $70{ }^{\circ} \mathrm{C}$, razão mola 1:15 do óleo de palma para o metanol e foi utilizado de 3 a $6 \%$ dos catalisadores.

Alguns autores já vêm estudando $\mathrm{KOH}(25 \%)$ suportado em $\mathrm{Al}_{2} \mathrm{O}_{3}$, como Ghasemi e Dehkordi (2014) que avaliou a reação de transesterificação de óleo de fritura na presença de $\mathrm{KOH}$ suportado em $\gamma-\mathrm{Al}_{2} \mathrm{O}_{3}$ utilizando o reator two-impinging-jet (TIJR). Na qual, foi obtido resultados melhores com o two-impinging-jet (TIJR) (76,35\%) quando comparado ao reator em batelada (58,01\%). As condições reacionais utilizadas foram de $4 \%$ de catalisador, razão molar óleo:metanol (1:15) e $65{ }_{-}^{\circ} \mathrm{C}$ de temperatura. Ilgen e Akin (2009) utilizaram $10 \% \mathrm{KOH}$ suportado em $\gamma-\mathrm{Al}_{2} \mathrm{O}_{3}$ na transesterificação de óleo de canola e obteve $89,40 \%$ de ésteres metílicos para as seguintes condições reacionais: temperatura de $60{ }^{\circ} \mathrm{C}$, razão molar do óleo para o metanol de 1:12, 3\% de catalisador e 9 horas de reação.

Para a determinação desses teores de ésteres são utilizadas diversas técnicas como, por exemplo, a cromatografia gasosa com detector de ionização de chama (ILGEN e AKIN , 2009), cromatografia gasosa com o detecto de massa, cromatografia líquida de alta eficiência (CLAE), ressonância magnética nuclear de hidrogênio e carbono ( $R M N^{1} \mathrm{H} \mathrm{e}^{13} \mathrm{C}$ ) e infravermelho próximo (IV) são alguns exemplos CMONTEIRO et. al, 2008; MEHER et. al, 2006; KNOTHE et. al, 2006). A norma EN14103 utiliza o detector de ionização de Chama. No entanto, Silva (2015) utilizou a 
termogravimetria (TG/DTG) para avaliar o teor de ésteres e acompanhar a reação de transesterificação de óleo de girassol por rota etílica.

Portanto, este trabalho tem como objetivo obter o catalisador e caracterizar, além de aplicá-lo na reação de transesterificação de óleo de algodão em busca das melhores condições de reações para obter a maior conversão de triglicerídeos em ésteres.

\section{METODOLOGIA}

\subsection{Preparação do catalisador $\left(\mathrm{KOH} / \mathrm{Al}_{2} \mathrm{O}_{3}\right)$}

O catalisador foi obtido pelo método de impregnação por excesso de solvente utilizando solução aquosa de hidróxido de potássio $(\mathrm{KOH})$ para ser suportado no em alumina $\left(\mathrm{Al}_{2} \mathrm{O}_{3}\right)$. $\mathrm{O}$ suporte $\left(\mathrm{Al}_{2} \mathrm{O}_{3}\right)$ foi pré-tratado a $80 \stackrel{\circ}{ } \mathrm{C}$ por $2 \mathrm{~h}$ em estufa para retirada de umidade. Depois foi armazenada em um dessecador até atingir a temperatura ambiente ( 25 ㅇ) para ser posteriormente submetida ao processo de impregnação. O procedimento de obtenção do material $\left(\mathrm{KOH} / \mathrm{Al}_{2} \mathrm{O}_{3}\right)$ foi realizado de acordo com Noiroj e colaboradores (2009), conforme demonstrado na Figura 1.

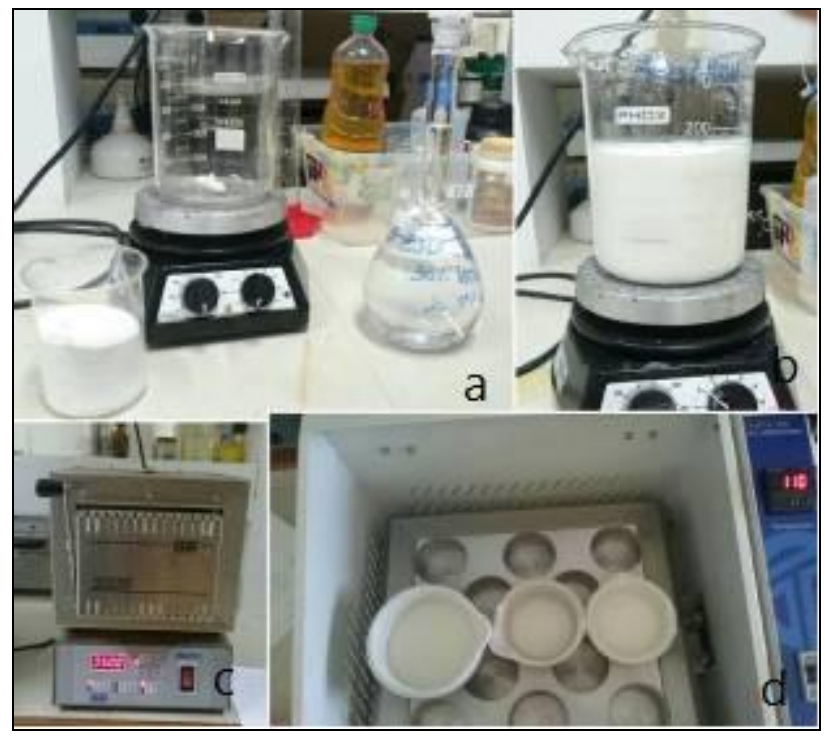

Figura 1: Processo de obtenção do catalisador $\left(\mathrm{KOH} / \mathrm{Al}_{2} \mathrm{O}_{3}\right)$ - Processo de impregnação (a e b), secagem (d) e calcinação (c).

Inicialmente, prepara-se uma solução de $30 \%$ de $\mathrm{KOH}$ (aquosa), em seguida $30 \mathrm{~mL}$ da mesma é misturado em $10 \mathrm{~g}$ de alumina, a mistura resultante foi mantida por cerca de $3 \mathrm{~h}$ sob aquecimento e agitação constante a 80 ․ Em seguida, a mistura foi transferida para um cadinho e levado a estufa para secagem a $110 \circ \mathrm{C}$ por $24 \mathrm{~h}$. Antes de ser aplicada a reação de transesterificação, o catalisador foi calcinado em um forno do tipo mufla em temperatura de 500 oC por $3 \mathrm{~h}$ em ar sintético sob fluxo de $100 \mathrm{~mL} \mathrm{~min}^{-1}$, conforme a representação esquemática do fluxograma da síntese está descrita na Figura 2. 


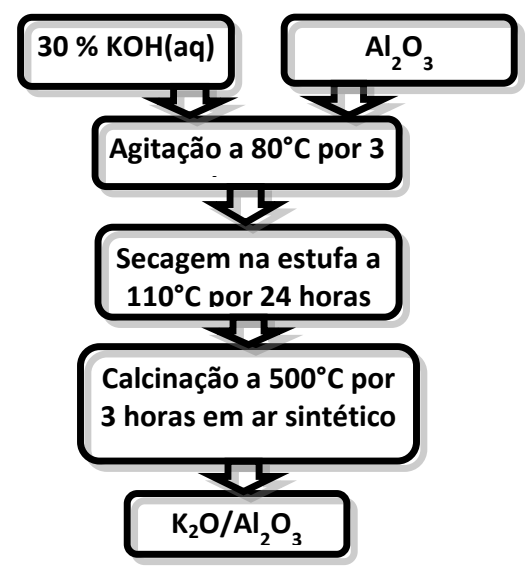

Figura 2: Fluxograma geral da síntese do catalisador $\left(\mathrm{KOH} / \mathrm{Al}_{2} \mathrm{O}_{3}\right)$.

\subsection{Caracterização do catalisador}

As caracterizações do catalisador foram realizadas utilizando as técnicas de difração de raios X (DRX), microscópio eletrônico de varredura acoplado com EDS (MEV-EDS), análise térmica (TG/DTG) e espectrometria na região do infravermelho por transformada de Fourier (FTIR).

Difração de raios $X$ é um método de caracterização utilizado para identificação da estrutura e de fases cristalinas. Os difratogramas de raios $X$ dos materiais foram obtidos em um equipamento da Shimadzu, modelo XRD 6000. Os dados foram coletados na faixa de $2 \theta$ de 10 a 80 graus com velocidade de goniômetro de $2 \stackrel{\circ}{\circ} \mathrm{min}^{-1}$ com um passo de 0,02 graus.

As análises de microscopia eletrônica de varredura acoplada com EDS (MEV - EDS) do catalisador foram realizadas em um equipamento Philipps modelo XL30-ESEM. Com o MEV podemos observa rapidamente informações sobre a morfologia e identificação de elementos químicos de uma amostra sólida. Outra característica importante é a aparência tridimensional da imagem das amostras, resultado direto da grande profundidade de campo.

As análises termogravimétricas (TG/DTG) para essas amostras foram realizadas com o uso de uma termobalança da Mettler Toledo TGA/SDTA, modelo 851e. As curvas TG foram obtidas utilizado cadinho de alumina com a faixa da temperatura ambiente até $900{ }^{\circ} \mathrm{C}$, com uma razão de aquecimento de $10{ }^{\circ} \mathrm{C} \mathrm{min}^{-1}$ e sob atmosfera de nitrogênio $\left(25 \mathrm{~mL} \cdot \mathrm{min}^{-1}\right)$.

A Espectroscopia na região do infravermelho por transformada de Fourier (FTIR) foram obtidos no modo transmitância no intervalo de 4000 a $400 \mathrm{~cm}^{-1}$ com resolução de $4 \mathrm{~cm}^{-1}$ e auxílio de uma janela de $\mathrm{KBr}$, em um espectrofotômetro de infravermelho com transformada de Fourier da Bomem, modelo MB102.

\subsection{Reação de transesterificação}

A reação de transesterificação foi realizada de acordo com Evangelista (2011) por rota etílica utilizando um sistema de refluxo aberto, com um balão de fundo chato de $500 \mathrm{~mL}$ 
conectado a um condensador, conforme Figura 3. A mistura reacional (óleo/etanol/catalisador) foi adicionada ao balão sob agitação e aquecimento constante de $70{ }^{\circ} \mathrm{C}$. As condições reacionais como a razão molar de etanol:óleo foi de (15:1) e a razão do catalisador foi de (4\%) onde foi variado o tempo de reação a fim de se obter um maior rendimento. Os tempos de reação estudados foram de $1 \mathrm{~h}$ (BIO 1h), 3h (BIO 3h) e 5h (BIO 5h).

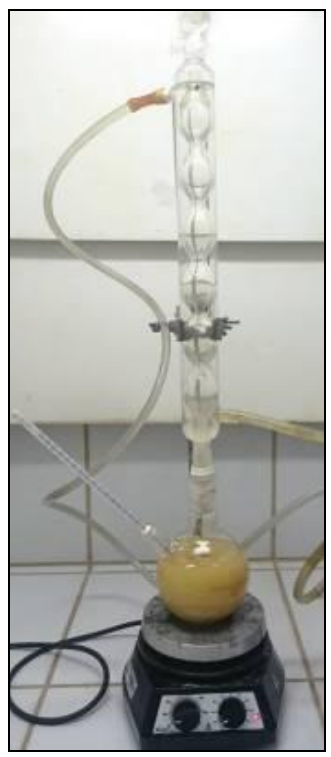

Figura 3: Sistema utilizado na reação de transesterificação.

Após terminar a reação, a mistura foi resfriada e separada por filtração para a remoção do catalisador. Em seguida, o filtrado foi transferido para um funil de decantação (Figura 4), formando duas fases (a fase superior constituída de ésteres etílicos impuros, e a fase inferior formada pelo glicerol e etanol). Após a retirada da glicerina, o biodiesel foi lavado com água e seco em estufa a $105^{\circ} \mathrm{C}$ por $3 \mathrm{~h}$.

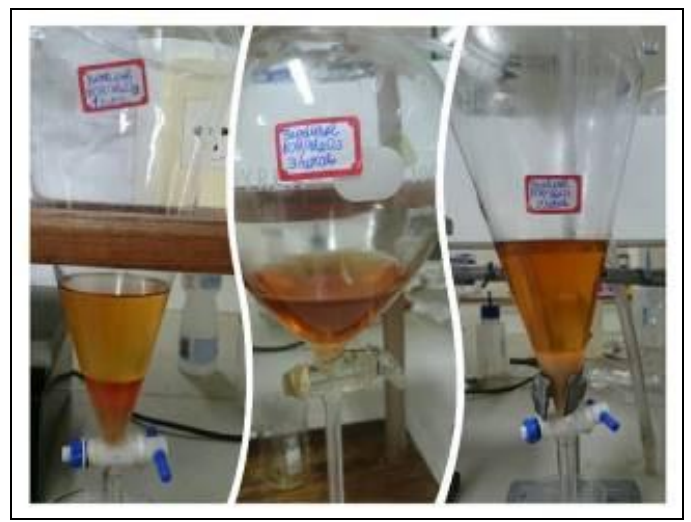

Figura 4: Separação da fase aquosa e dos ésteres etílicos (reações de 1 hora, 3 horas e 5 horas). 


\subsection{Caracterização do biodiesel}

O biodiesel obtido foi avaliado por densidade, viscosidade, análise térmica (TG/DTG) e espectroscopia de absorção na região do infravermelho por transformada Fourier (FTIR).

A densidade é um indicador de qualidade para combustíveis automotivos, de aviação e marítimos, afetando estocagem, manuseio e combustão. A análise foi realizada conforme metodologia da ASTM D4052, para um densímetro digital DMA4500 Anton Paar. As análises de viscosidade foram realizadas através de um viscosímetro de Stabinger SVM 3000 - Anton Paar.

A avaliação da reação de transesterificação foi realizada por termogravimetria (TG/DTG) de acordo com Silva (2015). As amostras de óleo de biodiesel de algodão foram analisadas usando uma termobalança Mettler Toledo TGA/SDTA, modelo 851e. As curvas termogravimétricas foram obtidas aquecendo cada uma das amostras, contidas em um cadinho de porcelana, na faixa da temperatura ambiente até $600 \stackrel{\circ}{\circ}$, com uma razão de aquecimento de $10{ }^{\circ} \mathrm{C} \mathrm{min}{ }^{-1}$ e sob atmosfera de nitrogênio com o fluxo de $25 \mathrm{~mL} \cdot \mathrm{min}^{-1}$. Através das curvas TG foi possível quantificar o teor de ésteres.

Espectros na região do infravermelho por transformada de Fourier (FTIR) foram obtidos através da técnica de Reflexão Total Atenuada - Attenuated Total Reflectance (ATR), utilizando a célula de ZnSe 45으, no modo transmitância no intervalo de 4000 a $700 \mathrm{~cm}^{-1}$ com resolução de 4 $\mathrm{cm}^{-1}$, em um espectrofotômetro de infravermelho com transformada de Fourier da Bomem, modelo MB102. O branco será realizado utilizando a célula de ZnSe sem amostra e para a obtenção dos espectros de ATR/FTIR será utilizado um volume suficiente para cobrir a célula. Através do FTIR é possível diferenciar os triglicerídeos dos ésteres obtidos por transesterificação.

\section{RESULTADOS E DISCUSSÕES}

\subsection{Caracterização do catalisador}

A Figura 5 mostra o difratograma de raios $\mathrm{X}$ do catalisador $\mathrm{KOH} / \mathrm{Al}_{2} \mathrm{O}_{3}$. Os padrões de $\mathrm{DRX}$ referentes à $\mathrm{Al}_{2} \mathrm{O}_{3}$ em $2 \theta=20^{\circ}, 32^{\circ}, 37^{\circ}, 46^{\circ}$ e $67^{\circ}$. A formação do catalisador $\mathrm{KOH} / \mathrm{Al}_{2} \mathrm{O}_{3}$ foi observada em $2 \theta=17^{\circ}, 23^{\circ}, 25^{\circ}, 29^{\circ}, 30^{\circ}, 31^{\circ}, 34^{\circ}, 38^{\circ}, 40^{\circ}, 44^{\circ}, 46^{\circ}, 47^{\circ}, 48^{\circ}, 51^{\circ}$ e $52^{\circ}$. Observase também o aparecimento de uma nova fase interpretada como sendo o $\mathrm{K}_{2} \mathrm{O}$ cujos picos podem ser observados para valores de $2 \theta$ em $51^{\circ}, 56^{\circ}, 69^{\circ}$ e $76^{\circ}$. 


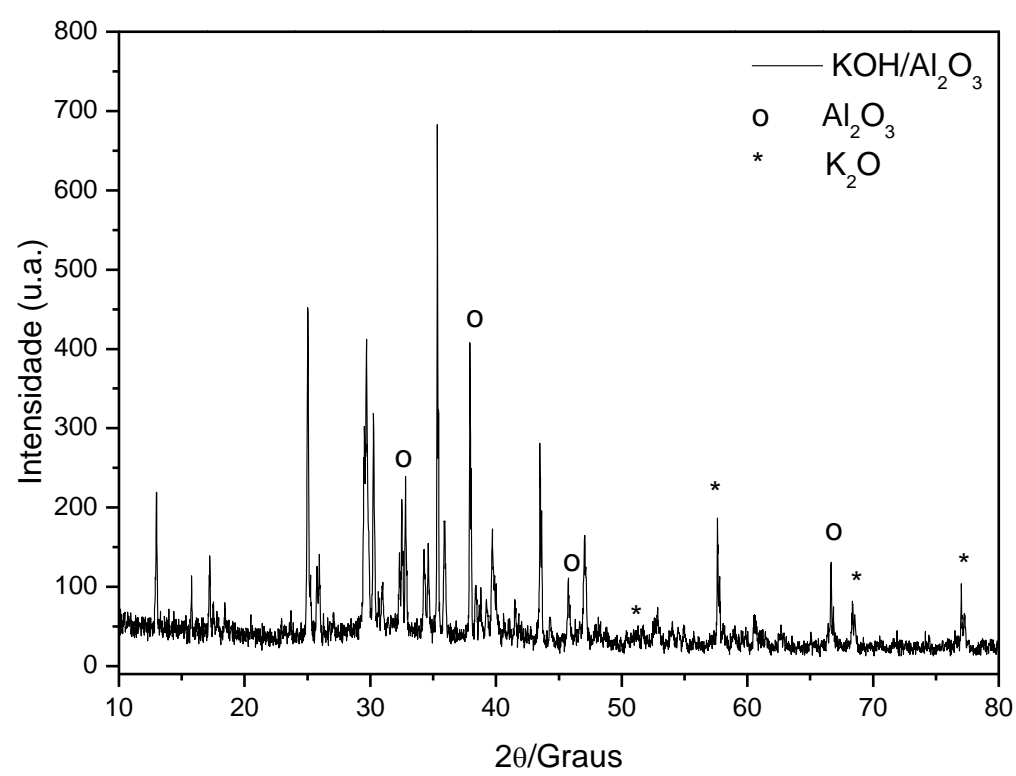

Figura 5: DRX do catalisador $\mathrm{KOH} / \mathrm{Al}_{2} \mathrm{O}_{3}$

As imagens do MEV-EDS estão apresentadas na Figura 6. Através do MEV observaram-se os aspectos morfológicos dos minerais e materiais. O MEV proporciona nítidas imagens e o EDS permite sua imediata identificação. Com o MEV-EDS, foi possível determinar a composição química pontual das fases minerais que compõem o catalisador, constituindo o EDS (Tabela 1) ferramenta indispensável na caracterização e distribuição espacial de elementos químicos.

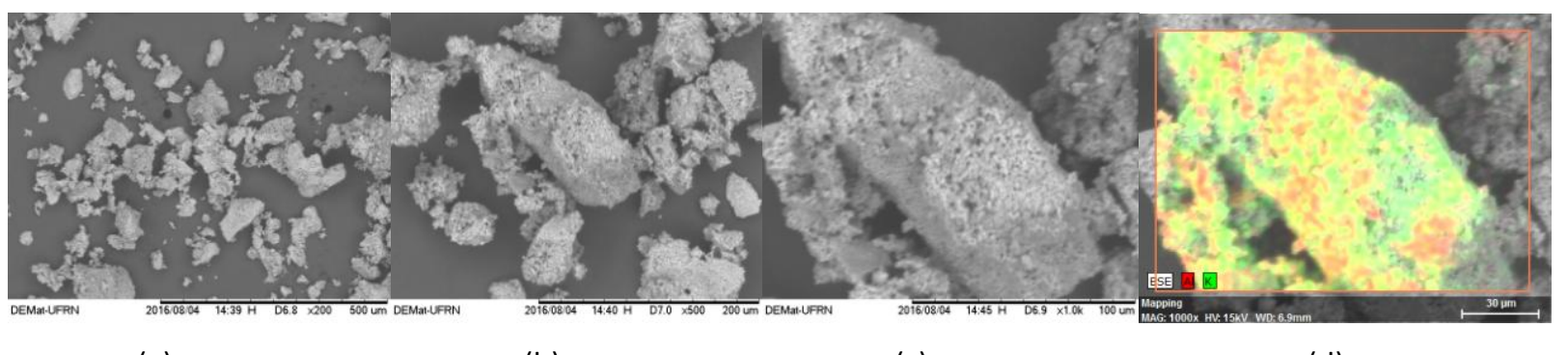

(a)

(b)

(c)

(d)

Figuras 6: Microscopia eletrônica de varredura da amostra do catalisador $\mathrm{KOH} / \mathrm{Al}_{2} \mathrm{O}_{3}$ (a) 100x, (b) 500x, (c) e (d) 1000x.

Tabela 1: EDS da amostra do catalisador $\mathrm{KOH} / \mathrm{Al}_{2} \mathrm{O}_{3}$.

\begin{tabular}{c|c|c|c}
\hline Elemento & Peso \% & Peso \% $\boldsymbol{\sigma}$ & Atômico \% \\
\hline Oxigênio & 83,26 & 0,72 & 90,85 \\
Alumínio & 8,39 & 0,49 & 5,43 \\
Potássio & 8,35 & 0,59 & 3,72 \\
\hline
\end{tabular}

As análises termogravimétricas (TG/DTG) foram realizadas com o objetivo de observar o processo de decomposição do catalisador $\mathrm{KOH} / \mathrm{Al}_{2} \mathrm{O}_{3}$. A Figura 7 apresenta a curvas TG/DTG de $\mathrm{KOH}, \mathrm{Al}_{2} \mathrm{O}_{3}$ do catalisador $\mathrm{KOH} / \mathrm{Al}_{2} \mathrm{O}_{3}$. 

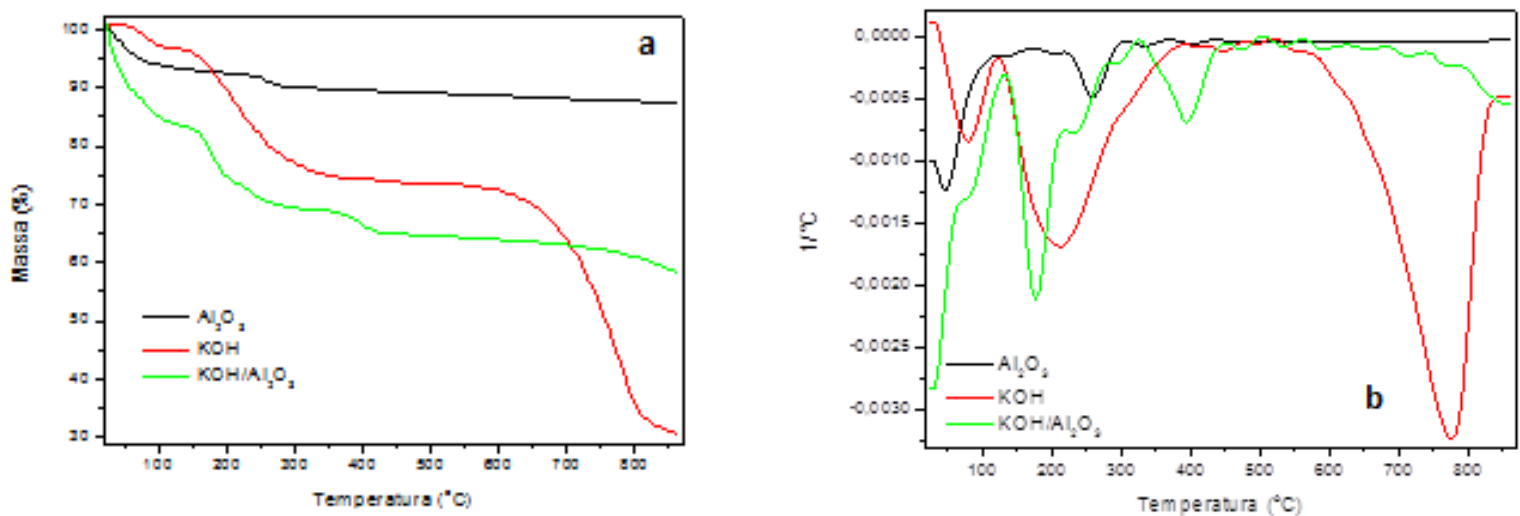

Figura 7: Curvas TG (a) e DTG (b) de $\mathrm{KOH}, \mathrm{Al}_{2} \mathrm{O}_{3}$ do catalisador $\mathrm{KOH} / \mathrm{Al}_{2} \mathrm{O}_{3}$.

A curva TG/DTG do $\mathrm{Al}_{2} \mathrm{O}_{3}$ apresenta duas perdas de massa referente à água fisissorvida e estrutural até $290{ }^{\circ} \mathrm{C}$, totalizado uma perda de massa total de $10 \% \mathrm{~m} / \mathrm{m}$. Para $\mathrm{O} \mathrm{KOH}$ foi observado três etapas de perda de massa onde a primeira e a segunda é referente à água fisissorvida e estrutural, e a terceira está relacionada à decomposição do $\mathrm{KOH}$. A decomposição térmica do catalisador $\left(\mathrm{KOH} / \mathrm{Al}_{2} \mathrm{O}_{3}\right)$ apresenta três etapas distintas. A primeira etapa $\left(132-217{ }^{\circ} \mathrm{C}\right.$, 34\%) corresponde à perda de água fisissorvida e a segunda referente à água estrutural, a terceira etapa com $13 \%$ de perda $\left(326-446^{\circ} \mathrm{C}\right)$ está associada à transformação do grupo $\mathrm{Al}-\mathrm{O}-\mathrm{H}$ no grupo $\mathrm{Al}-\mathrm{O}-\mathrm{K}$, juntamente com a formação das espécies $\mathrm{K}_{2} \mathrm{O} \cdot \mathrm{CO}_{2}(\mathrm{LI}, 2013)$.

Tabela 2: Dados obtidos da curva TG/DTG do catalisador $\mathrm{KOH} / \mathrm{Al}_{2} \mathrm{O}_{3}$.

\begin{tabular}{c|c|c|c}
\hline Amostra & Etapas & $\begin{array}{c}\text { Temperatura } \\
\text { (으) }\end{array}$ & $\begin{array}{c}\text { Perca de } \\
\text { massa (\%) }\end{array}$ \\
\hline $\mathrm{Al}_{2} \mathrm{O}_{3}$ & 1 & $30-100$ & 7 \\
& 2 & $200-290$ & 3 \\
\hline $\mathrm{KOH}$ & 1 & $45-120$ & 3 \\
& 2 & $120-350$ & 22 \\
& 3 & $530-825$ & 43 \\
\hline $\mathrm{KOH} / \mathrm{Al}_{2} \mathrm{O}_{3}$ & 1 & $132-217$ & 34 \\
& 3 & $326-446$ & 13 \\
\hline
\end{tabular}

Pode-se observar, na Figura 8, que a banda de absorção em $1385 \mathrm{~cm}^{-1}$ pode ser atribuída à ligação entre o potássio e oxigênio. A banda entre $3450-3700 \mathrm{~cm}^{-1}$ correspondem ao estiramento $(\mathrm{O}-\mathrm{H})$ que pode ser atribuída à água.

As bandas observadas na região próxima de $3500 \mathrm{~cm}^{-1}$ e próximas de $1635 \mathrm{~cm}^{-1}$ estão relacionadas, respectivamente, ao estiramento e flexão do grupo $\mathrm{OH}$ devido à presença de água adsorvida. As bandas que aparecem na região de $750-880 \mathrm{~cm}^{-1}$ envolvem estiramentos das ligações $\mathrm{Al}-\mathrm{O}$, uma vez que o alumínio apresenta vibrações por estiramento na região entre 750$850 \mathrm{~cm}^{-1}$ quando se encontram em coordenação tetraédrica (PEREIRA, 2016). 


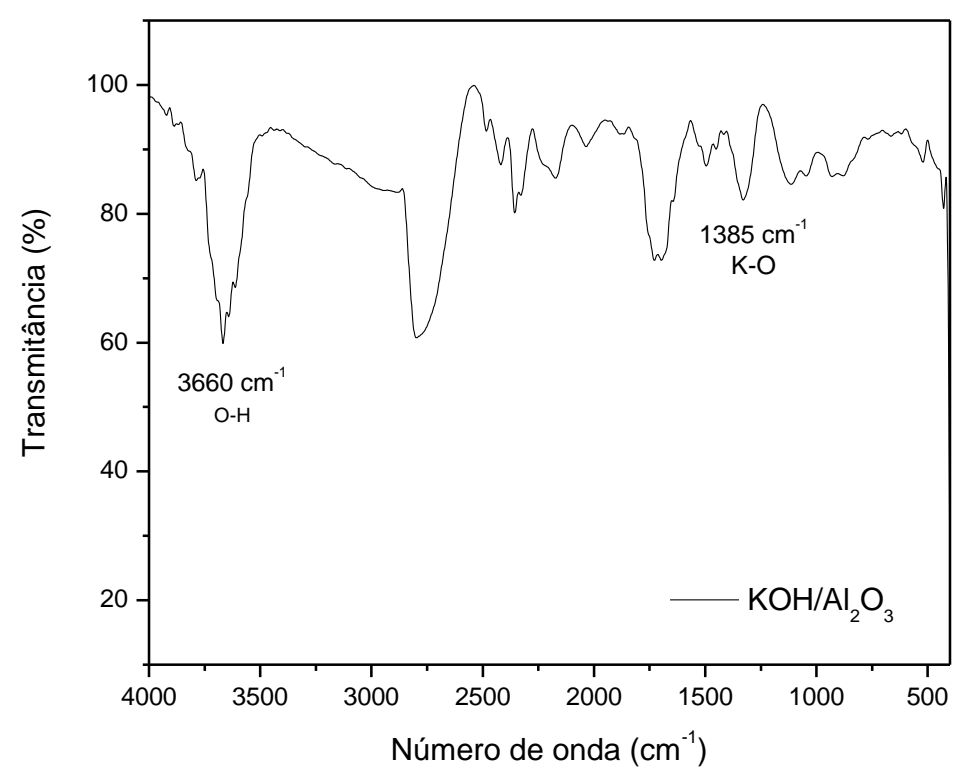

Figuras 8: $\mathrm{FTIR}$ da amostra do catalisador $\mathrm{KOH} / \mathrm{Al}_{2} \mathrm{O}_{3}$ após calcinação a $500{ }^{\circ} \mathrm{C}$.

\subsection{Caracterização do biodiesel de algodão}

A viscosidade é o parâmetro importante para a avaliação da reação de transesterificação, pois a viscosidade dos triglicerídeos (óleo) é reduzida quando convertidos em ésteres para faixa de $4-6 \mathrm{~mm}^{2} / \mathrm{s}$

É possível observar que os biodieseis obtidos apresentam redução na viscosidade cinemática à medida que foi aumentado o tempo reacional e principalmente com relação ao óleo de algodão que é de $33 \mathrm{~mm}^{2} / \mathrm{s}$. De acordo com o regulamento técnico ANP no 3/2014, as especificações da viscosidade cinemática para biodiesel devem estar entre $3-6$ pelo método ASTM D - 445. Para a qualidade do combustível a alta viscosidade provoca uma maior tendência de o combustível causar problemas na atomização do combustível durante a injeção na câmara de combustão (Tabela 3).

Tabela 3: Viscosidade das amostras de biodiesel de algodão à 40ㄷ.

\begin{tabular}{c|c|c}
\hline Biodiesel & $\begin{array}{c}\text { Viscosidade } \\
\text { dinâmica } \\
\text { (mPa.s) }\end{array}$ & $\begin{array}{c}\text { Viscosidade } \\
\text { cinemática } \\
\text { (mm } \mathbf{~} / \mathbf{s} \text { ) }\end{array}$ \\
\hline Óleo de & 30,253 & 33,406 \\
Algodão & & \\
BIO 1 h & 4,89 & 5,62 \\
BIO 3 h & 4,32 & 5,02 \\
BIO 5 h & 4,13 & 4,79 \\
\hline
\end{tabular}


Os resultados de densidade estão na Tabela 4. Observa-se que o aumento do tempo reacional promove diminuição na densidade do biodiesel. Este fato é um indicativo que quanto maior o tempo para a reação de transesterificação, uma maior massa de óleo é convertida em biodiesel, reduzindo assim a sua densidade.

Tabela 4: Densidade das amostras de biodiesel de algodão.

\begin{tabular}{c|c}
\hline Biodiesel & Densidade $\left(\mathrm{Kg} / \mathrm{m}^{3}\right)$ \\
\hline Óleo de Algodão & 905,6 \\
BIO 1 h & 882,6 \\
BIO 3 h & 882,2 \\
BIO 5 h & 877,2 \\
\hline
\end{tabular}

As análises termogravimétricas (TG/DTG) foram realizadas com o objetivo avaliar da reação de transesterificação através da diferença na temperatura de volatilização dos triglicerídeos e ésteres (SILVA et al., 2015). Assim, através dela foi possível observar o rendimento da reação de transesterificação, a fim de determinar o teor de ésteres totais (Figura 9).
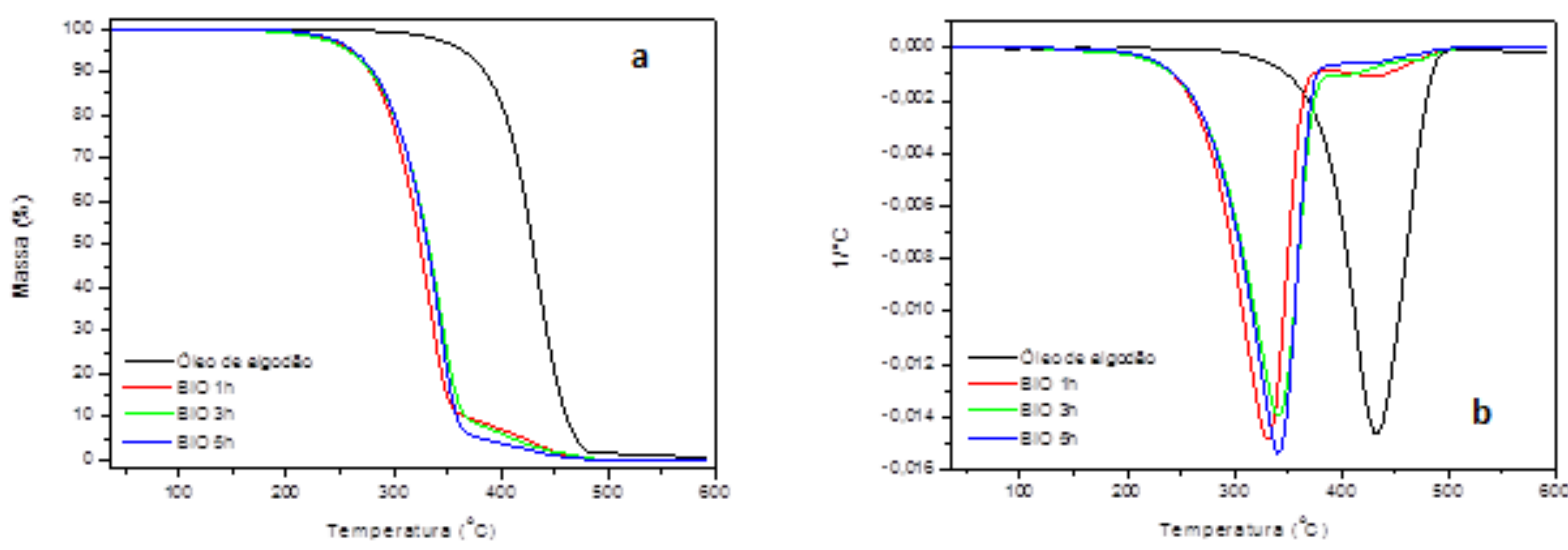

Figura 9: Curvas do TG (a) e DTG (b) biodiesel de algodão. BIO 1h - reação de 1 hora; BIO 3h - reação de 3 horas e BIO $5 \mathrm{~h}-5$ horas

A curva TG do óleo de algodão mostrou uma única perda de massa de 96,2\% e uma temperatura inicial de 304 으, referente à volatilização/evaporação dos ácidos graxos (triglicerídeos). As temperaturas iniciais da decomposição térmica dos biodieseis (ésteres) são inferiores à temperatura inicial de decomposição térmica do óleo, cerca de $100{ }^{\circ} \mathrm{C}$. Para as curvas TG/DTG de biodiesel (Figura 10 e 11) observa-se claramente duas etapas de decomposição, a primeira referente a volatilização/decomposição dos ésteres etílicos e a segunda a volatilização/decomposição dos triglicerídeos. Através da análise térmica observou-se que a maior perda de massa $(94,5 \%)$ é para a reação de $5 \mathrm{~h}$. A perda de massa para reação de 1 e $3 \mathrm{~h}$ é de $89 \%$. 
As curvas TG dos biodieseis de algodão, rota etanol, apresentaram comportamentos diferentes no perfil termogravimétrico, os quais estão relacionados com a atividade do catalisador na reação de transesterificação. Através das curvas termogravimétricas, observou-se que é possível mostrar, qualitativamente, o comportamento térmico de amostras de biodiesel e óleo de algodão corroborando com os resultados obtidos na literatura (SILVA, 2015)

Através dos espectros é possível verificar as variações nas intensidades das bandas de absorbância características de ésteres e de ácidos graxos na região do infravermelho. Na Figura 10 apresenta os espectros de FTIR do óleo de algodão e os biodieseis obtidos neste trabalho (BIO 1h, BIO $2 \mathrm{~h}$ e BIO 3h).

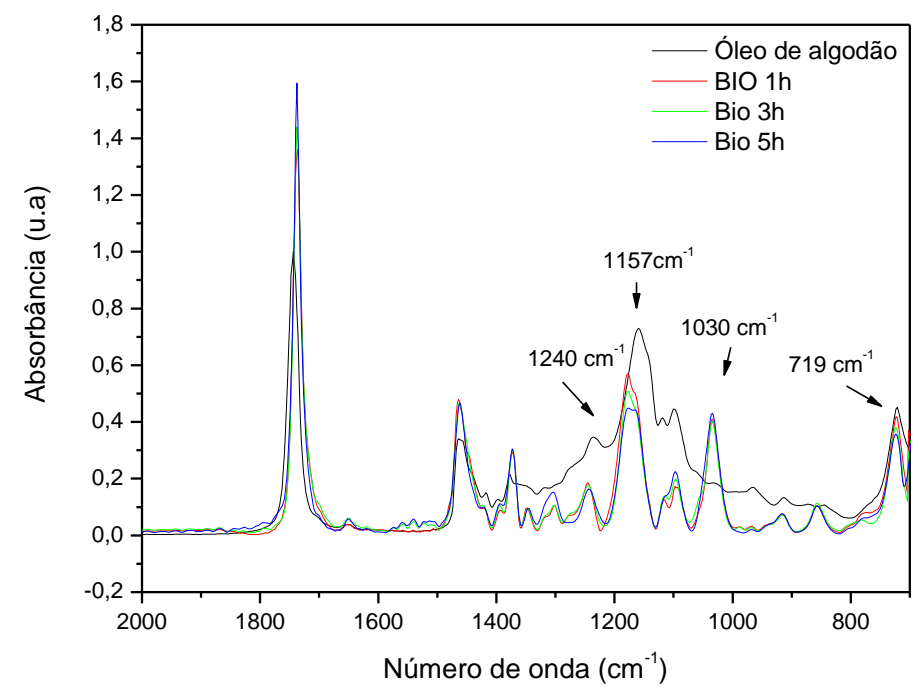

Figuras 10: FTIR do biodiesel de algodão. BIO $1 \mathrm{~h}$ - reação de 1 hora; BIO $3 \mathrm{~h}$ - reação de 3 horas e BIO $5 \mathrm{~h}-5$ horas

Através da figura pode-se observar que a banda de absorção em $1745,5 \mathrm{~cm}^{-1}$ pode ser atribuída ao grupo carbonila $(\mathrm{C}=\mathrm{O})$ e as bandas em 1455 e atribuídas à deformação angular do $\mathrm{CH}_{3}$ (SKOOG et al., 2002). A banda $719 \mathrm{~cm}^{-1}$, referente vibração de $-\mathrm{CH}_{2}$, e $1240 \mathrm{~cm}^{-1}$, referente à vibração da ligação de C-CO-O- e está presente para o óleo e o biodiesel. A banda $1157 \mathrm{~cm}^{-1}$ presente no óleo não está presente no biodiesel, mas surge duas bandas 1170 e $1160 \mathrm{~cm}^{-1}$. As absorções em 1370 e $1172 \mathrm{~cm}^{-1}$ são referentes a deformação $\mathrm{O}-\mathrm{CH}_{2}, \mathrm{O}-\mathrm{CH}_{3}$, respectivamente. Para o biodiesel ( esteres) surge a banda em $1030 \mathrm{~cm}^{-1}$.

\section{CONCLUSÃO}

Neste trabalho, o catalisador heterogêneo $\mathrm{KOH} / \mathrm{Al}_{2} \mathrm{O}_{3}$ foi sintetizado e caracterizado por diversas técnicas analíticas, na qual se confirmou a presença da fase ativa $\mathrm{K}_{2} \mathrm{O}$. Sua aplicação na reação de transesterificação do óleo de algodão foi considerada eficaz, pois apresentou redução na viscosidade e densidade que nos indica que ocorreu a conversão de triglicerídeos para ésteres. $\mathrm{Na}$ análise de infravermelho apresentou bandas características de ésteres (biodiesel) e ácidos graxos (trigliceridios), sendo possível diferenciar o óleo que foi transesterificado. $A$ análise 
termogravimétrica mostou que o biodiesel produzido no tempo reacional de $5 \mathrm{~h}$ apresentou maior percentual de conversão de triglicerídeos para ésteres (94,5\%).

\section{REFERÊNCIAS}

Agência Nacional de Petróleo, Gás Natural e Biocombustíveis - ANP. (2016) Disponível em: http://www.anp.gov.br/pg=17680\&m=5\%.

Boz, N.; Degirmenbasi, N.; Kalyon, D. (2009) Conversion of biomass to fuel: Transesterification of vegetable oil to biodiesel using KF loaded nano-gamma- $\mathrm{Al}_{2} \mathrm{O}_{3}$. Catalys Applied Catalysis B-Environmental, 89(3-4), 590-596.

Cordeiro C. S.; Silva F. R.; Wypych F.; Ramos L. P. (2011) Catalisadores heterogêneos para a produção de monoésteres graxos (biodiesel). Química Nova, 34 - 3, 477-486.

Evangelista, J. P. C. (2011) Obtenção de biodiesel através da transesterificação do óleo de farelo de arroz utilizando $\mathrm{KI} / \mathrm{Al}_{2} \mathrm{O}_{3}$. Natal, 2011. Dissertação de Mestrado - Universidade Federal do Rio Grande do Norte, Programa de Pós Graduação em Química.

Gama P. E. ; Lachtera E. R.; Gila R. A. S. S.; Coelho A. V.; Sidia I. A.; Poubela R. L.; Junior A. Da C. F.; Souza A. L. F. (2015) Caracterização e atividade catalítica de $\mathrm{K}_{2} \mathrm{CO}_{3} / \mathrm{Al}_{2} \mathrm{O}_{3}$ na transesterificação do óleo de girassol com aquecimento convencional e micro-ondas. Química Nova, 38 - 2, 185-190.

Ghasemi M.; Dehkordi A. M. (2014) Transesterification of Waste Cooking Oil to Biodiesel Using $\mathrm{KOH} / g a m m a-A l 2 \mathrm{O}$. Catalyst in a new two-impinging-jets reactor industrial \& engineering chemistry research, 53, $12238-12248$.

Gondim, A. D. (2009) Avaliação da estabilidade térmica e oxidativa do biodiesel de algodão e do efeito da adição de antioxidantes ( $\alpha$-tocoferol e BHT). Tese de Doutorado - Universidade Federal do Rio Grande do Norte, Programa de Pós Graduação em Química.

Ilgen O.; Akin A. N. (2009) Development of Alumina Supported Alkaline Catalysts Used for Biodiesel Production. Turkish journal of chemistry, 33, 281 -287.

Li, X.; Yu, D.; Zhang, W.; Li, Z.; Zhang, X.; Huang, H. (2013) Effective synthesis of cis-3-hexen-1-yl acetate via transesterification over $\mathrm{KOH} / \mathrm{Y}^{-}-\mathrm{Al}_{2} \mathrm{O}_{3}$. Structure and catalytic performance, Applied Catalysis A: General, 455, C,1-7

Knothe, G. (2006) Analyzing biodiesel: standards and other methods. Journal of the American Oil Chemists' Society, 83, 823-833.

Matos, P. R. R. (2011) Utilização de óleos vegetais como bases lubrificantes. Dissertação de Mestrado - Universidade de Brasília, Programa de Pós Graduação em Química.

Meher, L. C.; Sagar, D. V.; Naik, S. N. (2006) Technical aspects of biodiesel production by transesterification-a review. Renew Sustain. Energy Rev, 10, 248-268.

Monteiro, M. R.; Ambrozin, R. P.; Lião, L. M.; Ferreira, A. G. (2008) Critical review on analytical methods for biodiesel characterization. Talanta, 77, 593-605.

Mothé, C. G.; Azevedo, A. D. (2009) Análise térmica de materiais. Artliber. 
Noiroj, K.; Intarapong, P.; Luengnaruemitchai, A.; Jai-In, S. (2009) A comparative study of $\mathrm{KOH} / \mathrm{Al}_{2} \mathrm{O}_{3}$ and $\mathrm{KOH} / \mathrm{NaY}$ catalysts for biodiesel production via transesterification from palm oil. Renewable Energy 34, 1145-1150.

Oliveira, S. (2014) Biodiesel. Revista virtual de química, 6, 1021-1032.

Silva, V. (2015) Biodiesel e Biocombustível: Como fazer biodiesel? Disponível em: http://valterbio.blogspot.com.br/.

Oliveira, S. M. M.; Costa, B., J. (2006) Produção de biodiesel. Dossiê técnico, Instituto de Tecnologia do Paraná.

Olutoye, M. A.; Hameed, B.H. (2013) Production of biodiesel fuel by transesterification of different vegetable oils with methanol using $\mathrm{Al}_{2} \mathrm{O}_{3}$ modified $\mathrm{MgZnO}$ catalyst. Bioresource Technology, 132, 103-108.

Santos, L. K.; Cestari, A. Santos, L. K.; Cestari, A. (2014) Aluminato de potássio: um catalisador sintético e reutilizável para uso na produção de biodiesel. Revista virtual de química, 6, 10211032.

Silva, J. C. T.; Gondim, A. D.; Galvão, L. P. F. C.; Evangelista, J. P. C.; Araujo, A. S.; Júnior V. J. F. (2015) Thermal stability evaluation of biodiesel derived from sunflower oil obtained through heterogeneous catalysis $\left(\mathrm{KNO}_{3} / \mathrm{Al}_{2} \mathrm{O}_{3}\right)$ by thermogravimetry. J Therm Anal Calorim, 119, 715720.

Umdu, E. S.; Seker, E. (2012) Transesterification of sunflower oil on single step sol-gel made $\mathrm{Al}_{2} \mathrm{O}_{3}$ supported $\mathrm{CaO}$ catalysts: effect of basic strength and basicity on turnover frequency. Bioresource technology, 106, 178-81.

Xie, W.; Li, H. (2006) Alumina-supported potassium iodide as a heterogeneous catalyst for biodiesel production from soybean oil. Journal of Molecular Catalysis A: Chemical, 255, 1-9.

Yan, S.; Salley, S. I.; Ng, K. Y S. (2009) Oil transesterification over calcium oxides modified with lanthanum. Applied Catalysis A, 353, 163-170. 